\title{
Clinical manifestations in patients with SOS1 mutations range from Noonan syndrome to CFC syndrome
}

\author{
Yoko Narumi · Yoko Aoki · Tetsuya Niihori · Masahiro Sakurai · Hélène Cavé $\cdot$ \\ Alain Verloes · Kimio Nishio · Hirofumi Ohashi · Kenji Kurosawa · Nobuhiko Okamoto • \\ Hiroshi Kawame · Seiji Mizuno $\cdot$ Tatsuro Kondoh · Marie-Claude Addor · Anne Coeslier-Dieux • \\ Catherine Vincent-Delorme · Koichi Tabayashi - Masashi Aoki - Tomoko Kobayashi · \\ Afag Guliyeva $\cdot$ Shigeo Kure $\cdot$ Yoichi Matsubara
}

Received: 23 April 2008/Accepted: 23 June 2008/Published online: 24 July 2008

(C) The Japan Society of Human Genetics and Springer 2008

\begin{abstract}
Noonan syndrome (NS) and cardio-facio-cutaneous (CFC) syndrome are autosomal dominant disorders characterized by heart defects, facial dysmorphism, ectodermal abnormalities, and mental retardation. There is a significant clinical overlap between NS and CFC syndrome, but ectodermal abnormalities and mental retardation are
\end{abstract}

Y. Narumi · Y. Aoki $(\bowtie) \cdot$ T. Niihori · T. Kobayashi ·

A. Guliyeva $\cdot$ S. Kure $\cdot$ Y. Matsubara

Department of Medical Genetics,

Tohoku University School of Medicine,

1-1 Seiryo-machi, Sendai 980-8574, Japan

e-mail: aokiy@mail.tains.tohoku.ac.jp

M. Sakurai · K. Tabayashi

Department of Cardiovascular Surgery, Tohoku University

Graduate School of Medicine, Sendai, Japan

H. Cavé · A. Verloes

Department of Genetics, Hôpital Robert Debré

(APHP), Paris, France

K. Nishio

Department of Clinical Genetics, Seirei Hamamatsu

General Hospital, Hamamatsu, Japan

K. Nishio

Nishio Family Clinic, Hamamatsu, Japan

H. Ohashi

Division of Medical Genetics, Saitama Children's

Medical Center, Saitama, Japan

K. Kurosawa

Division of Medical Genetics, Kanagawa Children's

Medical Center, Yokohama, Japan

N. Okamoto

Department of Planning and Research,

Osaka Medical Center and Research Institute for Maternal and Child Health, Osaka, Japan more frequent in CFC syndrome. Mutations in PTPN11 and $K R A S$ have been identified in patients with NS and those in $K R A S, B R A F$ and $M A P 2 K 1 / 2$ have been identified in patients with CFC syndrome, establishing a new role of the RAS/MAPK pathway in human development. Recently, mutations in the son of sevenless gene (SOS1) have also

\section{H. Kawame}

Division of Medical Genetics, Nagano Children's Hospital,

Nagano, Japan

\section{S. Mizuno}

Department of Pediatrics, Central Hospital,

Aichi Human Service Center, Aichi, Japan

T. Kondoh

Division of Developmental Disability,

Misakaenosono Mutsumi Developmental,

Medical, and Welfare Center, Isahaya, Japan

M.-C. Addor

Department of Medical Genetics, CHU Vaudois,

Lausanna, Switzerland

A. Coeslier-Dieux

Department of Medical Genetics,

CHRU de Lille, Lille, France

C. Vincent-Delorme

$\mathrm{CH}$ d'Arras, Arras, France

M. Aoki

Department of Neurology,

Tohoku University Graduate School of Medicine,

Sendai, Japan 
been identified in patients with NS. To clarify the clinical spectrum of patients with SOS1 mutations, we analyzed 24 patients with NS, including 3 patients in a three-generation family, and 30 patients with CFC syndrome without PTPN11, KRAS, HRAS, BRAF, and MAP2K1/2 (MEK1/2) mutations. We identified two SOS1 mutations in four NS patients, including three patients in the above-mentioned three-generation family. In the patients with a CFC phenotype, three mutations, including a novel three amino-acid insertion, were identified in one CFC patient and two patients with both NS and CFC phenotypes. These three patients exhibited ectodermal abnormalities, such as curly hair, sparse eyebrows, and dry skin, and two of them showed mental retardation. Our results suggest that patients with SOS1 mutations range from NS to CFC syndrome.

Keywords PTPN11 - RAS - Noonan syndrome · Cardio-facio-cutaneous syndrome $\cdot \mathrm{RAF}$

\section{Introduction}

Noonan syndrome (NS; MIM\#163950) is an autosomaldominant developmental disorder characterized by facial dysmorphism, including hypertelorism, low-set ears, ptosis, short stature, skeletal abnormalities, and heart defects (Allanson et al. 1985; Mendez and Opitz 1985). Frequently observed features in NS patients are pulmonary stenosis (PS), hypertrophic cardiomyopathy, chest deformities, a webbed and short neck, mental retardation, genitourinary defects, including cryptorchidism in males, and bleeding diathesis due to factor XI deficiency (Mendez and Opitz 1985). The incidence of this syndrome is estimated to be 1 in 1,000-2,500 live births. NS has overlapping features with Costello syndrome and cardio-facio-cutaneous (CFC) syndrome. Patients with Costello syndrome show distinctive coarse facial features, mental retardation, high birth weight, neonatal feeding problems, curly hair, nasal papillomata, loose integuments on the back of the hands, and hypertrophic cardiomyopathy (Hennekam 2003). CFC syndrome is characterized by a distinctive face, mental retardation, heart defects [PS, atrial septal defect (ASD) and hypertrophic cardiomyopathy], ectodermal abnormalities, such as sparse, friable hair, hyperkeratotic skin lesions, and a generalized ichthyosis-like condition (Reynolds et al. 1986; Wieczorek et al. 1997).

The molecular pathogenesis of these syndromes has recently been elucidated. Tartaglia et al. have identified missense mutations in PTPN11, a gene encoding protein tyrosine phosphatase (PTP) SHP-2, in 45\% of clinically diagnosed NS patients (Tartaglia et al. 2001). In 2005, we discovered HRAS germline mutations in patients with Costello syndrome (Aoki et al. 2005). This finding was a clue for the identification of mutations in $K R A S, B R A F$, and $M A P 2 K 1 / 2$ in CFC syndrome (Niihori et al. 2006; Rodriguez-Viciana et al. 2006; Narumi et al. 2007). Mutations in $K R A S$ have also been identified in patients with NS (Schubbert et al. 2006). These findings suggest that dysregulation of the RAS/RAF/MEK/ERK pathway causes NS and related disorders, and thus it has been suggested that these syndromes be comprehensively termed the RAS/ MAPK syndromes (Aoki et al. 2008). Recently, gain-offunction mutations in the son of sevenless ( $\mathrm{SOS} 1$ ) gene have been identified in $17-21 \%$ of patients with NS (Roberts et al. 2007; Tartaglia et al. 2007). However, mutations in SOS1 have not been identified in patients with clinically diagnosed CFC syndrome, and the clinical spectrum of patients with SOS1 mutations has not been clarified.

In this study, we analyzed the SOS1 gene in 24 patients with NS and 30 patients with CFC syndrome without mutations in PTPN11, HRAS, KRAS, BRAF, and MAP2K1/ 2. Clinical manifestations in patients with SOS1 mutations were examined.

\section{Methods}

\section{Patients}

Twenty-one sporadic NS patients and three patients from a large family without PTPN11 mutations were recruited (Niihori et al. 2005) and this study). Also, 30 patients with CFC syndrome were recruited: 18 patients from the previous studies (Niihori et al. 2006; Narumi et al. 2007) and 12 newly referred patients with CFC syndrome. The first diagnoses by clinical geneticists were accepted. In SOS1-positive patients, clinical diagnoses were re-evaluated by clinical geneticists (K.N., H.O., and A.V.). Coding exons with flanking intronic sequences in $H R A S, K R A S, B R A F$, and $M A P 2 K 1 / 2$ were amplified and sequenced as previously described (Niihori et al. 2006; Narumi et al. 2007). No mutations were identified in any of the patients. Control DNA was obtained from 105 healthy Japanese individuals. Control DNA from 105 healthy Caucasian individuals was purchased from Coriell Cell Repositories. This study was approved by the Ethics Committee of Tohoku University School of Medicine. We obtained informed consent from all subjects involved in the study and specific consent for photographs from three patients from an NS family and two sporadic patients.

Mutation analysis in SOSI

Genomic DNA was isolated from the patients' peripheral leukocytes. Each exon with flanking intronic sequences in SOS1 was amplified with primers based on GenBank sequences (Table 1, GenBank accession no. NC_000002.10). 
Table 1 Primers used in this study

\begin{tabular}{|c|c|c|c|c|c|}
\hline Gene & Exon & Forward & Reverse & Product length (bp) & Annealing temperature $\left({ }^{\circ} \mathrm{C}\right)$ \\
\hline \multirow[t]{25}{*}{ SOS1 } & 1 & $5^{\prime}$-F-ctgttccgcgctgcgagc & $5^{\prime}$-R-gcagaccgggaagaaagacg & 319 & 56 \\
\hline & 2 & $5^{\prime}$-F-tgcccggccacaaacccac & $5^{\prime}$-R-ccctgttcactgacattacaacc & 323 & 58 \\
\hline & 3 & $5^{\prime}$-F-caggatgtgatattccccctag & $5^{\prime}$-R-ccttctcaccacataaatctctgg & 553 & 58 \\
\hline & 4 & $5^{\prime}$-F-gttggtaagcacaggcctc & $5^{\prime}$-R-ctggagatattccccaacac & 438 & 58 \\
\hline & 5 & $5^{\prime}$-F-cagagaacttagagcatttcac & $5^{\prime}$-R-gtgatcatgaattaagtcccac & 460 & 58 \\
\hline & 6 & $5^{\prime}$-F-tgcaaattgtacacctttgcag & $5^{\prime}$-R-agctggaaagaagtaagactc & 449 & 58 \\
\hline & $7 \& 8$ & $5^{\prime}$-F-tgtgctcgcatagtcgtgcc & $5^{\prime}$-R-cactaatgtgcagggtactcac & 512 & 58 \\
\hline & 9 & $5^{\prime}$-F-gcctgtcacaagatataaccc & $5^{\prime}$-R-gagggaactgggatcctg & 477 & 58 \\
\hline & $10 \mathrm{~A}$ & $5^{\prime}$-F-gtgaataccttctcagtgagac & $5^{\prime}$-R-ctcttcagctgacttggcag & 546 & 58 \\
\hline & 10B & $5^{\prime}$-F-ggcagccaagacttcctggtg & $5^{\prime}$-R-ggcacaataaacccatgcagg & 545 & 58 \\
\hline & 11 & $5^{\prime}$-F-gtccaaagcettctacttggc & $5^{\prime}$-R-gaaaaggatcttagctcaatctc & 324 & 58 \\
\hline & 12 & $5^{\prime}$-F-gactggtgaaaacgtttgtgg & $5^{\prime}$-R-ctccttgtttgggaaaggtcc & 328 & 58 \\
\hline & 13 & $5^{\prime}$-F-caaacaaggagaggggtgac & $5^{\prime}$-R-tactgagccccaatgacatc & 452 & 58 \\
\hline & 14 & $5^{\prime}$-F-tcaggtgtcatccgtgtgac & $5^{\prime}$-R-aaccctataaggcagaaatcag & 436 & 56 \\
\hline & 15 & $5^{\prime}$-F-gttttcacagacctttctgttgg & $5^{\prime}$-R-gacagagcaaaactccgtctc & 395 & 58 \\
\hline & 16 & $5^{\prime}$-F-ttatactatcgecacceccta & $5^{\prime}$-R-ctactgaaaagacaaatgag & 422 & 60 \\
\hline & 17 & $5^{\prime}$-F-gggcgtttctgttagcctag & $5^{\prime}$-R-ttctaaagggcttcaggtgc & 378 & 60 \\
\hline & 18 & $5^{\prime}$-F-ggcaactgagatggtacagtg & $5^{\prime}$-R-ctccctataaaataaacctgcc & 393 & 56 \\
\hline & 19 & $5^{\prime}$-F-ggcaggtttattttataggggag & $5^{\prime}$-R-ggaagtgggatattcctggac & 455 & 56 \\
\hline & $20 \mathrm{~A}$ & $5^{\prime}$-F-tagctgaattttaccaggcac & $5^{\prime}$-R-ctgtggtactggaagcaccag & 338 & 58 \\
\hline & $20 \mathrm{~B}$ & $5^{\prime}$-F-ccacacctctgcagcaggag & $5^{\prime}$-R-gttggtggagtttagaatttgtc & 392 & 58 \\
\hline & 21 & $5^{\prime}$-F-cttctcaaaagtaagtagtaa & $5^{\prime}$-R-gtaccaatgctgccagaccc & 347 & 59 \\
\hline & 22 & 5'-F-gtctgcatgcttttatggcag & 5'-R-gagaactaaactagacagccg & 415 & 56 \\
\hline & $23 \mathrm{~A}$ & $5^{\prime}$-F-tagcatcctgccaatagcatg & $5^{\prime}$-R-gtgccgtgaggagaaggtg & 436 & 56 \\
\hline & $23 \mathrm{~B}$ & $5^{\prime}$-F-ccaccacgagaacctgtgag & $5^{\prime}$-R-gctggcacattcagtgcatcc & 479 & 56 \\
\hline
\end{tabular}

For: 5' - gtaaaacgacggccagt Rev: 5'-aggaaacagctatgacc

The M13 reverse or forward sequence was added to the $5^{\prime}$ end of the polymerase chain reaction (PCR) primers for use as a sequencing primer. PCR was performed in $30 \mu \mathrm{l}$ of a solution containing $10 \mathrm{mM}$ Tris- $\mathrm{HCl}(\mathrm{pH} 8.3), 50 \mathrm{mM} \mathrm{KCl}, 1.5 \mathrm{mM}$ $\mathrm{MgCl}_{2}, 0.2 \mathrm{mM}$ dNTP, $10 \%$ (v/v) DMSO, 0.4 pmol of each primer, 100 ng genomic DNA, and 2.5 units of Taq DNA polymerase. The reaction condition consisted of 35 cycles of denaturation at $94^{\circ} \mathrm{C}$ for $15 \mathrm{~s}$, annealing at indicated temperatures for $15 \mathrm{~s}$, and extension at $72^{\circ} \mathrm{C}$ for $40 \mathrm{~s}$. The products were gel-purified and sequenced on an ABI PRISM 310 automated DNA sequencer (Applied Biosystems).

\section{Results}

Cinical manifestations in three-generation family

The pedigree of the NS family (NS13) is shown in Fig. 1. The proband of this pedigree NS13 (III-2) was found to have a systolic murmur at 29 years of age. She was diagnosed as having NS based on her facial appearance, including hypertelorism, ptosis, downslanting palpaebral fissures, and low-set ears. Webbed neck, cubitus valgus, and pectus deformity were not observed. Her height was $1.4 \mathrm{~m}(-2.5 \mathrm{SD})$, and she showed normal psychomotor development. Echocardiography of the heart revealed valvar PS and ASD. The patient underwent open-heart surgery for the PS and ASD. The proband's mother [NS 13 (II-6), 55 years old] and daughter [NS13 (IV-1), 5 years old] were found to have similar facial dysmorphisms, short stature, PS, and ASD. Both patients received open-heart surgery for PS and ASD. Chromosome analysis of NS13 (IV-1) showed a normal 46,XX karyotype. A younger sister of NS 13 (III-2) died soon after birth, but the cause of death was unknown. The proband's grandmother [NS 13 (I-1)] had a similar facial appearance with NS13 (II-6), but medical evaluations were not been performed. Other family members were clinically normal. There was no consanguinity in this family.

Mutation analysis in patients

We analyzed SOS1 in 24 patients with clinically diagnosed NS and identified two mutations in three patients from the 


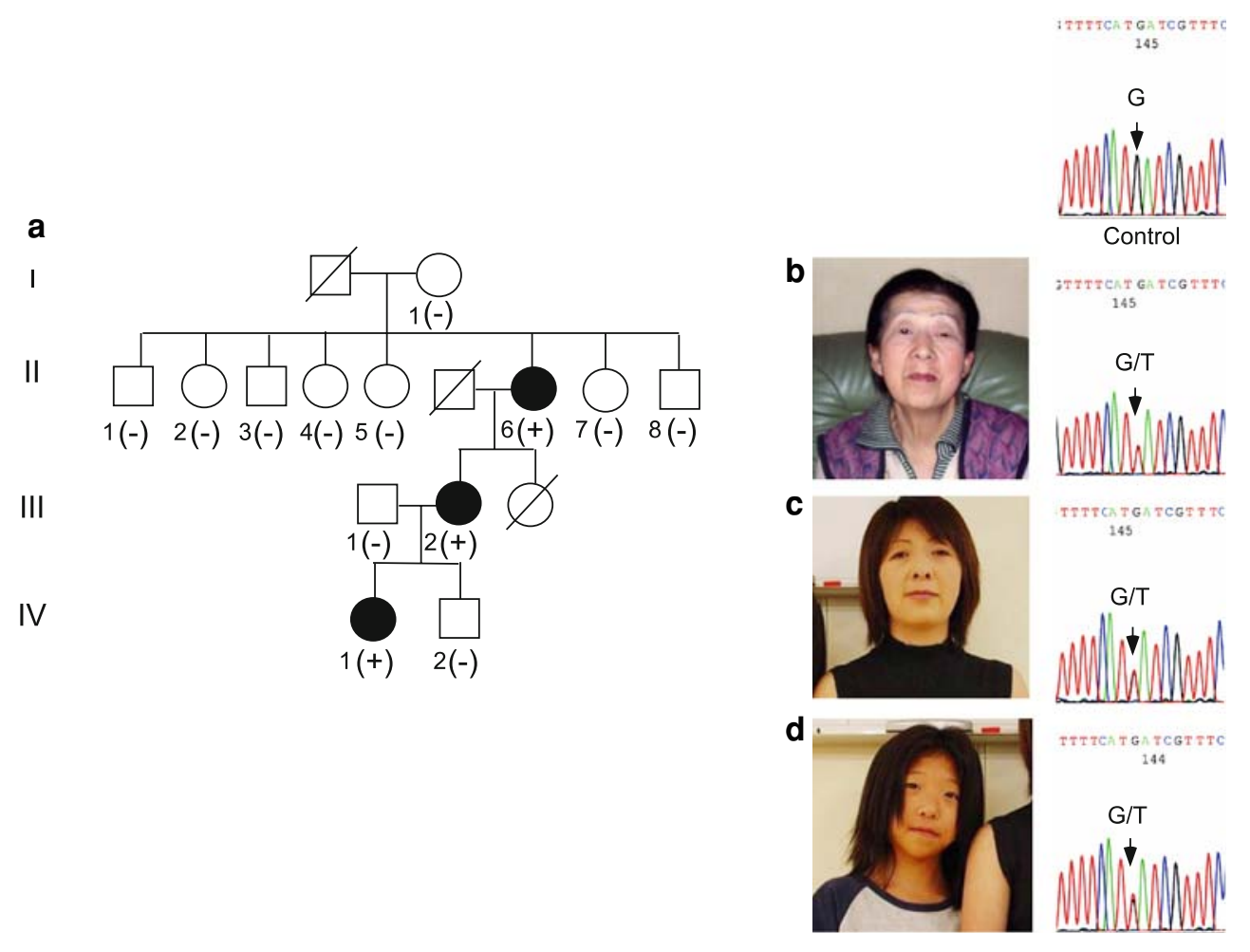

Fig. 1 The SOS1 D309Y mutation was identified in three patients in a family. a Pedigrees of the family. Square male, circle female, open symbol unaffected, filled symbol affected, + mutation positive, -

large family and one sporadic NS patient. In patient NS13 (III-2) in the large family, a $\mathrm{G}$ to $\mathrm{T}$ substitution at nucleotide 925 in SOS1, changing D309 to Y, was identified in the heterozygous form. This mutation was also identified in patients NS13 (II-6) and NS13 (IV-1), but not in ten other family members. The D309Y was not detected in 105 normal control individuals. We identified a c.2536G > A (E846 K) mutation in exon 17 in a patient with NS (NS45, Table 2).

From the group of 30 mutation-negative patients with CFC syndrome, we identified three heterozygous mutations in three patients: a c.1656G $>\mathrm{T}$ (R552S) mutation in NS 204, a novel in-frame 9-bp insertion in c.1442_1443 of exon 10 in NS84, and E846 K in NS 211 (Fig. 2). R552S was located in the pleckstrin homology (PH) domain and has been identified in four patients with NS (Fig 3) (Tartaglia et al. 2007; Zenker et al. 2007a). E846 K in the RAS guanine nucleotide exchange factor (GEF) catalytic domain has been identified in eight NS patients (Fig. 3) (Roberts et al. 2007; Tartaglia et al. 2007; Zenker et al. 2007a). The in-frame 9-bp insertion in c.1442_1443 of exon 10 resulted in three amino-acid insertion (P481_G482insRLP). The insertion was not detected in the patient's mother, but the father's DNA was not available. This insertion was not detected in 105 controls. A c.2999G $>$ A $(\mathrm{S} 1000 \mathrm{~N})$ mutation was identified in a patient with CFC syndrome. The substitution was identified in an unaffected mother and mutation-negative. Facial appearance and sequencing results of NS13 (II-6) (b), NS13 (III-2) (c), and NS13 (IV-1) (d). The positions of nucleotide substitutions are indicated

may represent SNP or a weak allele (Roberts et al. 2007; Tartaglia et al. 2007; Zenker et al. 2007a). A P655L, which has been reported as a SNP or a weak allele (Roberts et al. 2007; Tartaglia et al. 2007; Zenker et al. 2007a), was found in 3 out of 14 CFC patients from France.

Clinical manifestations of SOS1-positive patients

Three patients from the large family had similar facial appearances, PS, ASD, short stature, and normal psychomotor development. Patient NS45 was diagnosed as having NS because of her typical facial appearance, PS, pectus deformity, and normal mental development. Patient NS84 was recruited from original CFC patients (Niihori et al. 2006; Narumi et al. 2007). He has the CFC Gestalt, including round face, coarsening of facial traits, curly hair, and sparse eyebrows, as well as aortic valve stenosis and school delay (Table 2; Fig. 2). Patient NS211 is atypical both for NS and for CFC. He has unusually marked hypertelorism, a very short upturned nose with a wide large tip, and small ears (Fig. 2). He also has clinical features that clearly fit the NS to CFC spectrum, including down slanting of the palpebral fissures, ptosis, a deep philtral groove, and a coarse face. Skin and hair anomalies, including deep palmar creases and dry skin, as well as mental retardation resembled those of CFC patients. The 
Fig. 2 SOS1 mutations and clinical features in sporadic patients. a Sequencing result of PCR products from control DNA and genomic DNA of NS 211. The positions of nucleotide substitutions are indicated. Photos of NS211 at 6 months $(\mathbf{b}, \mathbf{c})$ and at 1 year of age $(\mathbf{d}, \mathbf{e})$. Facial appearance in NS211 with marked hypertelorism, very short upturned nose with a very large tip, and small ears. f Sequencing result of a PCR product from genomic DNA of NS84. The PCR product was subcloned and sequenced. The result showed that there is a 9bp insertion in one allele, resulting in the three aminoacids insertion. The 9-bp insertion is indicated as rectangles. Photos of NS211 at 4 years of age $(\mathbf{g}, \mathbf{h})$ and at 6 years of age $(\mathbf{i}, \mathbf{j})$. Clinical features in NS84 show coarsening of facial traits, curly hair, and sparse eyebrows
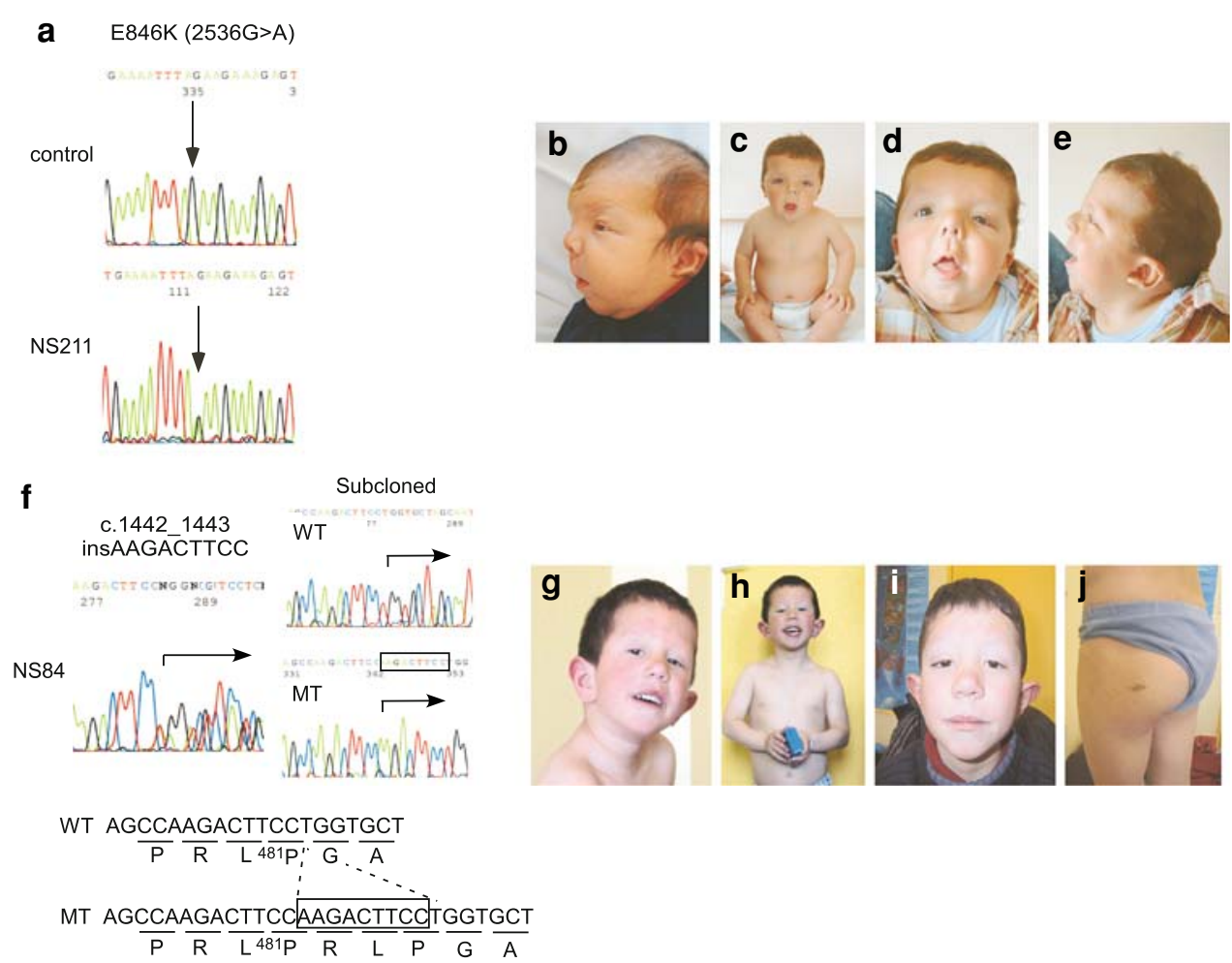
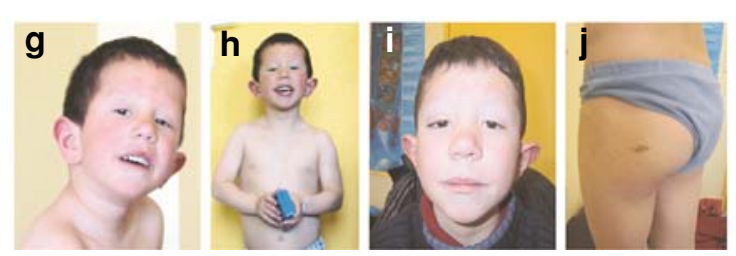

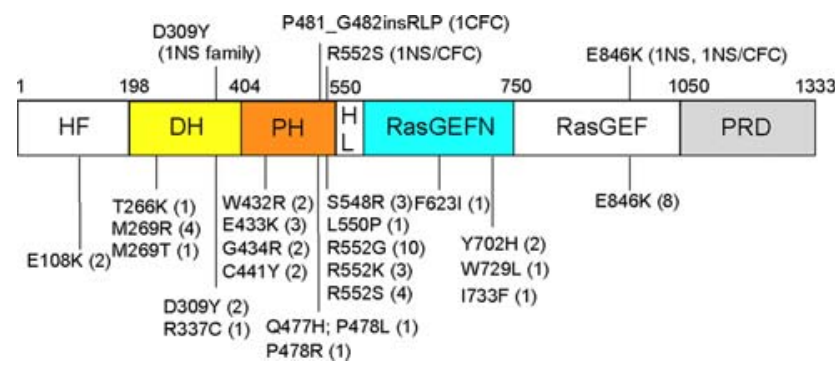

Fig. 3 Domain organization and identified mutations in the SOS1 gene. The functional domains are indicated as follows: $H F$ histonelike fold, $D H$ Dbl homology, $P H$ pleckstrin homology, $H L$ helical linker, REM Ras-exchange motif, RASGEF RAS guanine nucleotide exchange factor, $P R D$ proline-rich domains. Mutations detected in this study are shown above the domain structure, and mutations identified in the past studies (Roberts et al. 2007; Tartaglia et al. 2007; Zenker et al. 2007a) are shown below the domain structure

phenotype of patient NS204 was between NS and CFC in the early infantile period (1 year and 4 months). He has sparse hair and eyebrows, but this is not that remarkable in a very young child with fair hair. His face is rather large (more similar to CFC than NS).

\section{Discussion}

In this study, we identified a novel 3-amino-acid insertion and three missense mutations in SOS1 in 2 of $22(9 \%)$ families with NS and 3 of 30 (10\%) patients from the group with CFC syndrome. The mutation detection rate was lower than those reported in previous reports: 12/57 (21\%) of cases with NS without PTPN11, KRAS and BRAF mutations (Roberts et al. 2007), 22/129 (17\%) patients with NS without PTPN11 and KRAS mutations (Tartaglia et al. 2007), and $28 \%$ of patients with NS without mutations in PTPN11, KRAS, BRAF and MAP2K1/2 (Zenker et al. 2007a). The lower detection rate in our study might be due to the smaller size of our cohorts.

SOS1 mutations were identified in three patients with a CFC phenotype. This is in contrast with a previous report in which no mutations in SOS1 were identified in CFC patients (Zenker et al. 2007a). Clinical manifestations of NS and CFC syndrome have been found to overalp, but several differences have been reported. CFC patients with $K R A S, B R A F$, and MAP2K1/2 mutations have been found to show a high frequency of mental retardation (100\%), sparse, curly hair $(96 \%)$, and skin problems, including follicular keratosis $(60 \%)$ and hyperkeratosis $(56 \%)(\mathrm{Na}-$ rumi et al. 2007). In contrast, the rates of mental retardation (24-35\%) and skin problems (2-27\%) are lower in NS patients (Wieczorek et al. 1997). Our patient NS84 is compatible with CFC syndrome because of his facial appearance, sparse curly hair, and moderate mental retardation. It is noteworthy that he has aortic valve stenosis, which is atypical of NS (Abadir et al. 2007) and has not been reported in patients with SOS1 mutations (Roberts et al. 2007; Tartaglia et al. 2007; Zenker et al. 2007a). In 


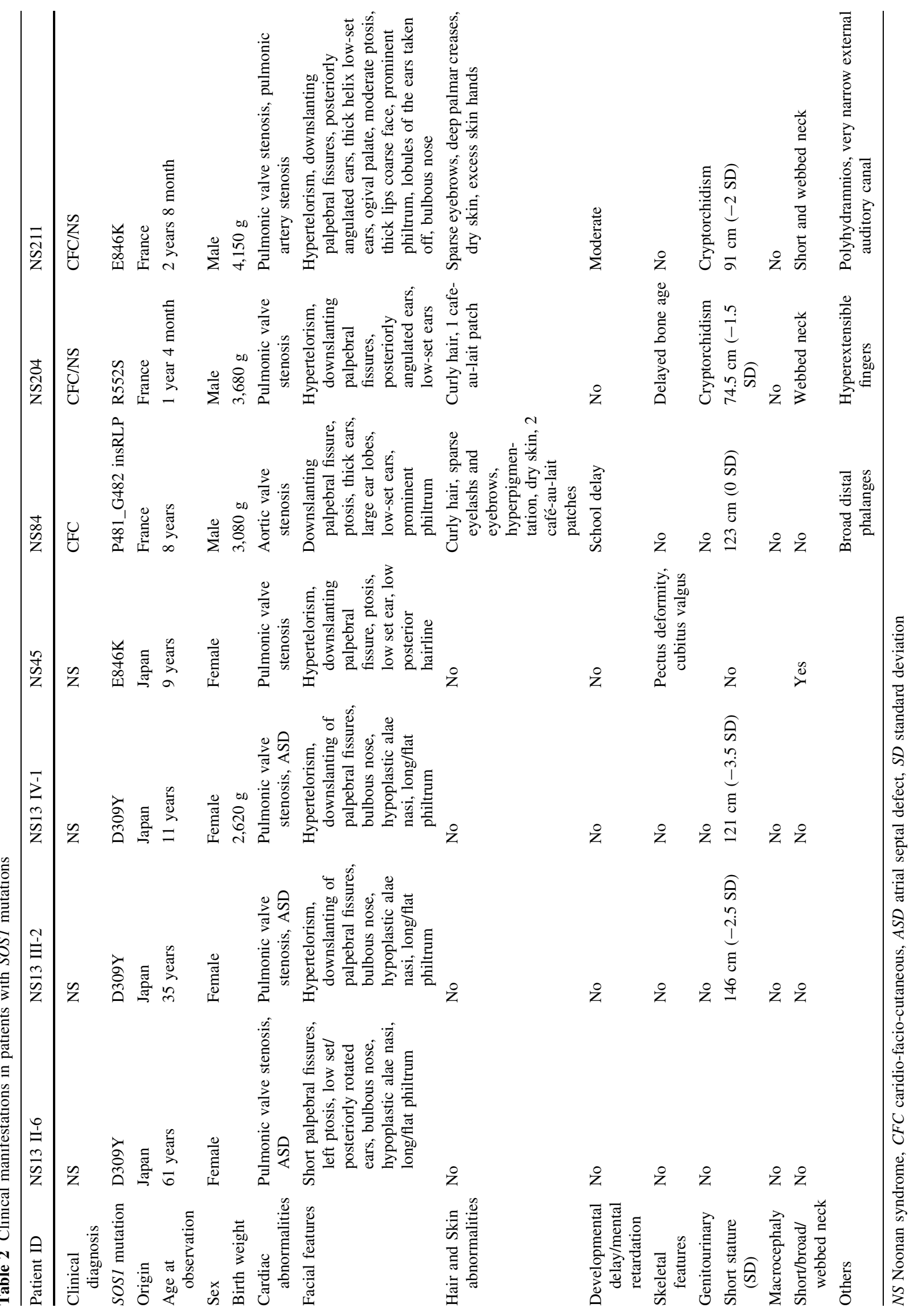


two other patients with a CFC/NS phenotype, ectodermal abnormalities are evident, including curly hair, sparse eyebrows, deep palmar creases, and dry skin. In a previous report, keratosis pilaris, curly hair, and macrocephaly in patients with SOS1 mutations were significantly more prevalent compared with typical NS or NS patients with PTPN11 mutations, suggesting that clinical manifestations of SOS1 mutations range from those typical of NS to those apparently resembling CFC syndrome (Tartaglia et al. 2007). Another group did not find SOS1 mutations in 21 CFC patients (Zenker et al. 2007a). Three patients from the NS group exhibited skin manifestations resembling those shown in CFC syndrome, but they were diagnosed as having NS because of normal psychomotor and mental development (Zenker et al. 2007a). Given that the clinical manifestations of NS and CFC syndrome overlap, it is not surprising that SOS1 mutations were identified in patients diagnosed as having CFC syndrome. Our results suggest that clinical manifestations in patients with SOS1 mutations range from NS to CFC syndrome.

All of the SOS1 mutations reported in NS patients have been missense mutations (Roberts et al. 2007; Tartaglia et al. 2007; Zenker et al. 2007a). We identified a novel 3amino-acid insertion in the PH domain in patient NS84 with a CFC phenotype. SOS1 is a ubiquitously expressed GEF, which is responsible for activation of RAS proteins by catalyzing GDP/GTP exchange (Bouguski and McCormick 1993). SOS1 is a $150-\mathrm{kDa}$ protein that has $\mathrm{Dbl}$ homology $(\mathrm{DH})$ and pleckstrin homology $(\mathrm{PH})$ domains in addition to a conserved catalytic region and a putative RAS-binding domain (Fig. 3). Approximately $50 \%$ of SOS1 mutations identified in patients were located in the PH domain (15/29, Fig. 3). This domain has been shown to play an important role in intramolecular regulation. The DH-PH domain has an autoinhibitory effect against catalytic activity of the RAS guanine nucleotide exchange factor (cdc25) (Roberts et al. 2007; Tartaglia et al. 2007). The R552G mutation in the PH domain is the most common mutation in NS patients (Fig.3) and has been shown to cause a significant increase of RAS activation and phosphorylation of ERK (Roberts et al. 2007; Tartaglia et al. 2007). Another function of the PH domain is to target the proteins to the plasma membrane though interactions with specific phospholipids (Soisson et al. 1998). The novel three amino-acid insertion (P481_G482insRLP) identified in this study is located in $\beta 3-\beta 4$ loop in the PH domain. Two other mutations (477H:P478L and P478R) in the $\beta 3$ $\beta 4$ loop of the PH domain have been identified in two sporadic patients with NS (Zenker et al. 2007a). The pocket between the $\beta 1-\beta 2$ and $\beta 3-\beta 4$ loops is not likely to affect the autoinhibitory effect (Sondermann et al. 2004), but potentially serves as the binding site of inositol $(1,4,5)$ triphosphate (Soisson et al. 1998). Recently, the conserved basic amino acids in the $\mathrm{PH}$ domain including $\mathrm{P} 481$ and G482 have been shown to have high affinity and specificity to phosphatidic acid (Zhao et al. 2007). The interaction between the PH domain and phosphatidic acid has been found to be essential for EGF-induced SOS membrane recruitment and Ras activation (Zhao et al. 2007). Structural changes caused by the three amino-acid insertion at P481 would alter the membrane translocation of SOS or Ras activation.

We identified the D309Y mutation in three patients of a family with NS. These patients had very similar clinical manifestations: facial dysmorphisms, short stature, ASD, PS, and normal psychomotor development. Although other skeletal abnormalities, including webbed neck, cubitus valgus, and chest deformity, were not evident, the features satisfied the clinical criteria of NS described by van der Burgt (van der Burgt et al. 1994). Two sporadic patients with a D309Y mutation have been reported (Roberts et al. 2007; Zenker et al. 2007a). Clinical variability among the affected members in a family has been reported in families with NS. Van der Burgt et al. reported clinical heterogeneity in a three-generation Dutch family with NS (van der Burgt et al. 1994). Seven of nine patients in the family showed PS, but two had no congenital heart anomalies. Seven had typical faces, and three had pectus carinatus/ excavatum. Linkage analysis of this family revealed that a gene for NS was located on 12q24.1, which is the location of PTPNII (Jamieson et al. 1994). Schollen et al. reported a four-generation NS family with a PTPN11 Q79R mutation (Schollen et al. 2003). The patients in this family showed either NS or CFC phenotype. T411 M and G409A mutations in PTPN11 were identified in two-generation families with clinical variability (Bertola et al. 2004; Zenker et al. 2007b). These results suggested that NS families with PTPN11 mutations exhibit clinical variability. Clinical manifestations in eight families with SOSI mutations have not been described (Roberts et al. 2007; Zenker et al. 2007a). The present report is the first of a large family having a SOS1 mutation with minimal clinical variability among affected family members. Our findings indicate that clinical manifestations may be similar among family members in a family with a SOSI mutation.

In conclusion, we identified four SOSI mutations in a three-generation family and four sporadic patients ranging from NS to CFC phenotype. Clinical manifestations in mutation-positive patients revealed that three patients exhibited ectodermal abnormalities and two had mental retardation. These abnormalities have been frequently observed in patients with CFC syndrome. Mutations in SOS1 were identified in only $10 \%$ of our patients with NS and CFC syndrome. Presently unknown genetic causes for mutation-negative NS and related disorders, including recently identified mutations in RAF-1 (Pandit et al. 2007; 
Razzaque et al. 2007), remain to be identified in molecules in the RAS/MAPK cascade.

Acknowledgments The authors wish to thank the patients and their families who participated in this study. We are grateful to Giovanni Neri, Maria Ines Kavamura, Raoul CM Hennekam, Gabriele Gillessen-Kaesbach, Dagmar Wieczorek, and Pablo Lapunzina, who referred the patients. We are grateful to Kumi Kato and Miyuki Tsuda for their technical assistance. We also thank Nathalie Pouvreau for her help in data management. This work was supported by Grants-inAid from the Ministry of Education, Culture, Sports, Science and Technology of Japan and a grant from Astellas Foundation for Research on Metabolic Disorders.

\section{References}

Abadir S, Edouard T, Julia S (2007) Severe aortic valvar stenosis in familial Noonan syndrome with mutation of the PTPN11 gene. Cardiol Young 17:95-97

Allanson JE, Hall JG, Hughes HE, Preus M, Witt RD (1985) Noonan syndrome: the changing phenotype. Am J Med Genet 21:507514

Aoki Y, Niihori T, Kawame H, Kurosawa K, Ohashi H, Tanaka Y, Filocamo M, Kato K, Suzuki Y, Kure S, Matsubara Y (2005) Germline mutations in HRAS proto-oncogene cause Costello syndrome. Nat Genet 37:1038-1040

Aoki Y, Niihori T, Narumi Y, Kure S, Matsubara Y (2008) The RAS/ MAPK syndromes: novel roles of the RAS pathway in human genetic disorders. Hum Mutat [Epub ahead of print]

Bertola DR, Pereira AC, de Oliveira PS, Kim CA, Krieger JE (2004) Clinical variability in a Noonan syndrome family with a new PTPN11 gene mutation. Am J Med Genet A 130:378-383

Hennekam RC (2003) Costello syndrome: an overview. Am J Med Genet C Semin Med Genet 117:42-48

Jamieson CR, van der Burgt I, Brady AF, van Reen M, Elsawi MM, Hol F, Jeffery S, Patton MA, Mariman E (1994) Mapping a gene for Noonan syndrome to the long arm of chromosome 12. Nat Genet 8:357-360

Mendez HM, Opitz JM (1985) Noonan syndrome: a review. Am J Med Genet 21:493-506

Narumi Y, Aoki Y, Niihori T, Neri G, Cave H, Verloes A, Nava C, Kavamura MI, Okamoto N, Kurosawa K, Hennekam RC, Wilson LC, Gillessen-Kaesbach G, Wieczorek D, Lapunzina P, Ohashi H, Makita Y, Kondo I, Tsuchiya S, Ito E, Sameshima K, Kato K, Kure S, Matsubara Y (2007) Molecular and clinical characterization of cardio-facio-cutaneous (CFC) syndrome: overlapping clinical manifestations with Costello syndrome. Am J Med Genet A 143:799-807

Niihori T, Aoki Y, Narumi Y, Neri G, Cave H, Verloes A, Okamoto N, Hennekam RC, Gillessen-Kaesbach G, Wieczorek D, Kavamura MI, Kurosawa K, Ohashi $\mathrm{H}$, Wilson L, Heron D, Bonneau D, Corona G, Kaname T, Naritomi K, Baumann C, Matsumoto N, Kato K, Kure S, Matsubara Y (2006) Germline KRAS and BRAF mutations in cardio-facio-cutaneous syndrome. Nat Genet 38:294-296

Niihori T, Aoki Y, Ohashi H, Kurosawa K, Kondoh T, Ishikiriyama S, Kawame H, Kamasaki H, Yamanaka T, Takada F, Nishio K, Sakurai M, Tamai H, Nagashima T, Suzuki Y, Kure S, Fujii K, Imaizumi M, Matsubara Y (2005) Functional analysis of PTPN11/SHP-2 mutants identified in Noonan syndrome and childhood leukemia. J Hum Genet 50:192-202

Pandit B, Sarkozy A, Pennacchio LA, Carta C, Oishi K, Martinelli S, Pogna EA et al (2007) Gain-of-function RAF1 mutations cause
Noonan and LEOPARD syndromes with hypertrophic cardiomyopathy. Nat Genet 39(8):1007-1012

Razzaque MA, Nishizawa T, Komoike Y, Yagi H, Furutani M, Amo R, Kamisago M, Momma K, Katayama H, Nakagawa M, Fujiwara Y, Matsushima M, Mizuno K, Tokuyama M, Hirota H, Muneuchi J, Higashinakagawa T, Matsuoka R (2007) Germline gain-of-function mutations in RAF1 cause Noonan syndrome. Nat Genet 39(8):1013-1017

Reynolds JF, Neri G, Herrmann JP, Blumberg B, Coldwell JG, Miles PV, Opitz JM (1986) New multiple congenital anomalies/mental retardation syndrome with cardio-facio-cutaneous involvement- the CFC syndrome. Am J Med Genet 25:413-427

Roberts AE, Araki T, Swanson KD, Montgomery KT, Schiripo TA, Joshi VA, Li L, Yassin Y, Tamburino AM, Neel BG, Kucherlapati RS (2007) Germline gain-of-function mutations in SOS1 cause Noonan syndrome. Nat Genet 39:70-74

Rodriguez-Viciana P, Tetsu O, Tidyman WE, Estep AL, Conger BA, Cruz MS, McCormick F, Rauen KA (2006) Germline mutations in genes within the MAPK pathway cause cardio-facio-cutaneous syndrome. Science 311:1287-1290

Schollen E, Matthijs G, Gewillig M, Fryns JP, Legius E (2003) PTPN11 mutation in a large family with Noonan syndrome and dizygous twinning. Eur J Hum Genet 11:85-88

Schubbert S, Zenker M, Rowe SL, Boll S, Klein C, Bollag G, van der Burgt I, Musante L, Kalscheuer V, Wehner LE, Nguyen H, West B, Zhang KY, Sistermans E, Rauch A, Niemeyer CM, Shannon K, Kratz CP (2006) Germline KRAS mutations cause Noonan syndrome. Nat Genet 38:331-336

Soisson SM, Nimnual AS, Uy M, Bar-Sagi D, Kuriyan J (1998) Crystal structure of the Dbl and pleckstrin homology domains from the human Son of sevenless protein. Cell 95:259-268

Sondermann H, Soisson SM, Boykevisch S, Yang SS, Bar-Sagi D, Kuriyan J (2004) Structural analysis of autoinhibition in the Ras activator Son of sevenless. Cell 119:393-405

Tartaglia M, Mehler EL, Goldberg R, Zampino G, Brunner HG, Kremer H, van der Burgt I, Crosby AH, Ion A, Jeffery S, Kalidas K, Patton MA, Kucherlapati RS, Gelb BD (2001) Mutations in PTPN11, encoding the protein tyrosine phosphatase SHP-2, cause Noonan syndrome. Nat Genet 29:465-468

Tartaglia M, Pennacchio LA, Zhao C, Yadav KK, Fodale V, Sarkozy A, Pandit B, Oishi K, Martinelli S, Schackwitz W, Ustaszewska A, Martin J, Bristow J, Carta C, Lepri F, Neri C, Vasta I, Gibson K, Curry CJ, Siguero JP, Digilio MC, Zampino G, Dallapiccola B, Bar-Sagi D, Gelb BD (2007) Gain-of-function SOS1 mutations cause a distinctive form of Noonan syndrome. Nat Genet 39:75-79

van der Burgt I, Berends E, Lommen E, van Beersum S, Hamel B, Mariman E (1994) Clinical and molecular studies in a large Dutch family with Noonan syndrome. Am J Med Genet 53:187-191

Wieczorek D, Majewski F, Gillessen-Kaesbach G (1997) Cardiofacio-cutaneous (CFC) syndrome-a distinct entity? Report of three patients demonstrating the diagnostic difficulties in delineation of CFC syndrome. Clin Genet 52:37-46

Zenker M, Horn D, Wieczorek D, Allanson J, Pauli S, van der Burgt I, Doerr HG, Gaspar H, Hofbeck M, Gillessen-Kaesbach G, Koch A, Meinecke P, Mundlos S, Nowka A, Rauch A, Reif S, von Schnakenburg C, Seidel H, Wehner LE, Zweier C, Bauhuber S, Matejas V, Kratz CP, Thomas C, Kutsche K (2007a) SOS1 is the second most common Noonan gene but plays no major role in cardio-facio-cutaneous syndrome. J Med Genet 44:651-656

Zenker M, Voss E, Reis A (2007b) Mild variable Noonan syndrome in a family with a novel PTPN11 mutation. Eur J Med Genet $50: 43-47$

Zhao C, Du G, Skowronek K, Frohman MA, Bar-Sagi D (2007) Phospholipase D2-generated phosphatidic acid couples EGFR stimulation to Ras activation by Sos. Nat Cell Biol 9:706-712 